\section{Is allopurinol a potential new treatment for angina pectoris?}

Peter Higgins', Matthew R Walters' \& Jesse Dawson ${ }^{\dagger 1}$

'Division of Cardiovascular \& Medical Sciences, Faculty of Medicine, University of Glasgow, Glasgow, UK tAuthor for correspondence: Tel.: +44 1412116395 m Fax: +44 1412112895 m j.dawson@clinmed.gla.ac.uk

Evaluation of: Noman A, Ang DSC, Ogston S, Lang CC, Struthers AD: Effect of high-dose allopurinol on exercise in patients with chronic stable angina: a randomized, placebo controlled crossover trial. Lancet 375, 2161-2167 (2010). Xanthine oxidase, the enzyme responsible for the final steps of uric acid production, is increasingly recognized as a contributor to oxidative stress in the vasculature. Furthermore, a potential role for uric acid in the pathogenesis of cardiovascular disease has been debated for decades. Inhibition of this enzyme by allopurinol is a licensed and commonly used strategy for the prevention of gout and recent data suggest it may also have beneficial effects on cardiovascular function. Perhaps the most compelling data to date were recently published in the Lancet by Noman and colleagues. They showed that allopurinol may be a novel anti-ischemic agent for the treatment of angina pectoris. We discuss this study and its wider implications to cardiovascular medicine in this article.

\section{Summary of background \& methods}

Experimental data in both animals and humans indicate that allopurinol reduces oxygen demand in the myocardium [1], probably through a multifactorial mechanism. Accordingly, the authors sought to evaluate whether allopurinol would prolong exercise capacity in those with chronic stable angina and thus serve as a novel anti-ischemic agent [2].

They studied 60 patients with angina pectoris, defined as coronary artery disease confirmed on angiography with reproducible ST-segment change on exercise tolerance testing (ETT). This well-characterized group of patients with stable but symptomatic angina were enrolled into a doubleblind randomized placebo-controlled crossover study. Allopurinol $600 \mathrm{mg}$ daily or placebo were each given for a period of 6 weeks in an order determined by randomization. Established antecedent anti-anginal therapy continued unchanged during the study. Bruce protocol ETT evaluation was conducted at baseline and again after each treatment phase. Although there was no 'washout' between treatment phases or reassessment of 'baseline' ETT prior to commencing the second treatment phase, appropriate consideration of a treatment order effect was made. The primary end point was change in time to demonstrable ST-depression, Secondary end points included total exercise time and time to development of typical angina symptoms. Although the sample size was small, it was based on an appropriate power calculation.

Results \& new information from this work In the first treatment period, 31 patients were allocated to allopurinol and 34 to placebo. Of those patients who were given allopurinol, 28 completed the first phase. Of those patients who were given placebo, 32 completed the first phase. These 60 patients all completed the second phase of study.

The time to median ST-depression was increased to $298 \mathrm{~s}$ following allopurinol treatment compared with $249 \mathrm{~s}$ following placebo (from a baseline of $232 \mathrm{~s}$, giving a difference between treatments of 43 s [ $95 \%$ CI: 31-58 s]). Total exercise time was also increased (from a baseline of $301 \mathrm{~s}$ to $307 \mathrm{~s}$ with placebo and to 393 s with allopurinol [95\% CI for the difference: $45-77 \mathrm{~s}]$ ). Allopurinol also increased the time to chest pain $(95 \%$ CI for the difference: $17-55 \mathrm{~s})$. Thus, on a variety of measures, allopurinol improved exercise capacity in patients with angina pectoris. While small improvement was seen following the placebo phase of the study (as might be expected with an end point of this nature), there was a far greater improvement following allopurinol. The authors also reported a reduction in circulating brain natriuretic peptide levels and (although it did not reach statistical significance), there was a trend toward a reduction in the frequency of anginal attacks and usage of as required nitrate therapy.

Importantly, these improvements were seen in the context of robust antecedent anti-anginal therapy, with greater than $85 \%$ of patients established on a $\beta$-blocker and approximately $50 \%$ treated with a nitrate prior to enrollment. No adverse effects were reported in association with treatment. The authors therefore concluded that allopurinol may now be considered a viable option for the management of patients with angina pectoris.
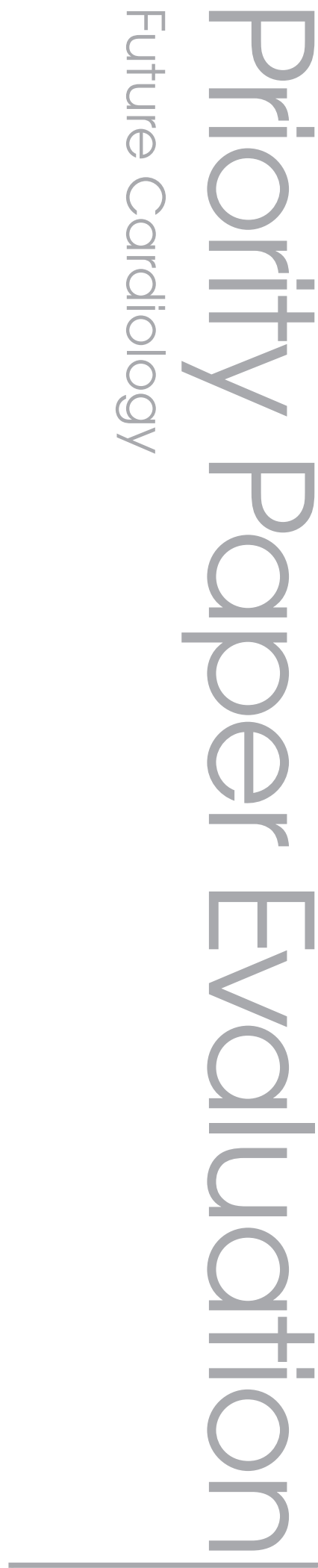

\section{Keywords}

- allopurinol = angina

- endothelium $=$ uric acid

= xanthine oxidase

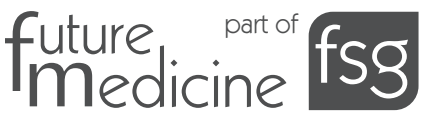




\section{Significance of these findings \& future} direction of research

The findings from this well-conducted study are no doubt encouraging. An additional antiischemic agent with a novel mechanism of action could improve symptom control in the large numbers with angina. Allopurinol appears to have similar efficacy to other more established treatment options and importantly, appears to add to them when used in combination. Taken together with the generally acceptable side effect profile and relatively low cost of allopurinol, this strategy must be further pursued.

Adverse reactions following allopurinol can be severe although no adverse drug reactions were observed in this study and the safety record for allopurinol in the treatment of gout is considered good. For example, we suggest it is at least comparable to that of antiplatelet therapy. It is important to note that this study utilized highdose treatment (600 mg daily), a significantly greater dose than is typical for gout prophylaxis. However, $600 \mathrm{mg}$ per day is still within the maximum licensed dose and while the potential for toxicity must be considered, these concerns should not preclude further evaluation. These concerns would be greatly offset if allopurinol were shown to reduce cardiovascular morbidity and mortality and there is potential for this.

The potential mechanism for the effect seen in this study is interesting. No change in the heart rate-blood pressure product was seen and it seems implausible that treatment led to regression of hemodynamically significant coronary stenoses. Furthermore, although hyperuricemia is linked with increased vascular mortality, it is not likely that lowering serum uric acid will have an anti-anginal effect. The observed effects are most likely due to inhibition of the xanthine oxidase enzyme itself and an ensuing effect on oxygen metabolism/consumption. Xanthine oxidase activity is a source of oxidative stress in the vasculature [3] and this may be particularly the case in areas of ischemia - perhaps challenging traditional wisdom that xanthine oxidase is of secondary importance compared with nicotinamide adenine dinucleotide phosphate-oxidase. Thus, by reducing oxidative stress, allopurinol may improve endothelial function [4], reduce the requirement of the myocardium for metabolic substrate and lower oxygen demand on exertion. This appears to translate into clinical benefit or in those with stable angina.

Allopurinol has also been shown to have a favorable effect on peripheral endothelial function [5], cerebrovascular nitric oxide bioavailability [6] and to reduce circulating markers of inflammation [7], to reduce blood pressure in a cohort of adolescents with recently diagnosed hypertension [8] and improve myocardial contractility [9]. Although the change in serum uric acid per se may not underlie the effect seen in this study, it is possible that it may bring further ancillary benefits in the longer term. Hyperuricemia may lead to increases in vascular smooth muscle proliferation and may increase low density lipoprotein oxidation [10]. Lowering serum uric acid may reduce development of further atherosclerotic lesions. Thus, as well as reducing symptoms of angina, there is a very real possibility that allopurinol will reduce cardiovascular event rates. Importantly, the level of serum uric acid could also serve as a surrogate marker for the degree of enzyme inhibition following allopurinol; as a biomarker to help titrate dose and ensure the optimal degree of enzyme inhibition is achieved.

We hope this high-profile publication will serve to facilitate future definitive studies [11]. At present, allopurinol is a promising novel treatment that has not yet been shown to be of benefit on hard clinical end points. Definitive recommendations regarding the broader application of this treatment strategy are difficult to make but will hopefully come in the near future.

\section{Conclusion}

The results from Noman and colleagues are encouraging on a number of levels. In practical terms they suggest promise toward a therapeutic advancement in the treatment of patients with angina pectoris. In addition, the findings add to a growing body of data indicating the benefit of xanthine oxidase inhibition on numerous measures of cardiovascular function. Xanthine oxidase inhibition with allopurinol is acceptably safe and may well reduce vascular event rates in those at highest risk. We look forward to further studies designed to confirm or refute this undoubted potential.

Financial \& competing interests disclosure

The authors have no relevant affiliations or financial involvement with any organization or entity with a financial interest in or financial conflict with the subject matter or materials discussed in the manuscript. This includes employment, consultancies, honoraria, stock ownership or options, expert testimony, grants or patents received or pending, or royalties.

No writing assistance was utilized in the production of this manuscript. 


\section{Executive summary}

- Allopurinol represents a novel theraputic strategy for the treatment and prevention of cardiovascular disease.

- Such benefit may be conferred through either uric acid reduction or reduced oxidative stress.

- The work of Noman and colleagues demonstrates that allopurinol improves exercise tolerance and symptoms in patients with stable anginal pectoris.

- Large clinical trials addressing definitive cardiovascular end points are indicated.

\section{Bibliography}

1. Cappola TP, Kass DA, Nelson GS et al.: Allopurinol improves myocardial efficiency in patients with idiopathic dilated cardiomyopathy. Circulation 104, 2407-2411 (2001).

2. Noman A, Ang DS, Ogston S, Lang CC, Struthers AD: Effect of high-dose allopurinol on exercise in patients with chronic stable angina: a randomized, placebo controlled crossover trial. Lancet 375, 2161-2167 (2010).

3. Landmesser U, Spiekermann S, Dikalov $S$ et al: Vascular oxidative stress and endothelial dysfunction in patients with chronic heart failure: role of xanthine-oxidase and extracellular superoxide dismutase. Circulation 106, 3073-3078 (2002).

4. George J, Carr E, Davies J, Belch JJ, Struthers A: High-dose allopurinol improves endothelial function by profoundly reducing vascular oxidative stress and not by lowering uric acid. Circulation 114, 2508-2516 (2006).

5. Yiginer O, Ozcelik F, Inanc T et al.: Allopurinol improves endothelial function and reduces oxidant-inflammatory enzyme of myeloperoxidase in metabolic syndrome. Clin. Res. Cardiol. 97, 334-340 (2008).

6. Dawson J, Quinn TJ, Harrow C et al: Allopurinol and nitric oxide activity in the cerebral circulation of those with diabetes. Diabetes Care 32, 135-137 (2009).

7. Muir S, Harrow C, Dawson J et al.: Allopurinol use yields potentially beneficial effects on inflammatory indices in those with recent ischemic stroke; a randomized, double blind placebo controlled trial. Stroke 39, 3303-3307 (2008).

8. Feig DI, Soletsky B, Johnson RJ: Effect of allopurinol on blood pressure of adolescents with newly diagnosed essential hypertension: a randomized trial. JAMA 300, 924-932 (2008).

9. Baldus S, Mullerleile K, Chumley P et al: Inhibition of xanthine oxidase improves myocardial contractility in patients with ischemic cardiomyopathy. Free Radic. Biol. Med. 41, 1282-1288 (2006).

10. Descheerder IK, Vandekraay AMM, Lamers JMJ, Koster JF, Dejong JW, Serruys PW: Myocardial malondialdehyde and uric-acid release after short-lasting coronary occlusions during coronary angioplasty - potential mechanisms for free-radical generation. $A m$. J. Cardiol. 68, 392-395 (1991).

11. Higgins P, Dawson J, Walters MR: The potential for xanthine oxidase inhibition in the prevention of cardiovascular and cerebrovascular disease. Cardiovasc. Psychiatry. Neurol. (2009) (Epub ahead of print). 